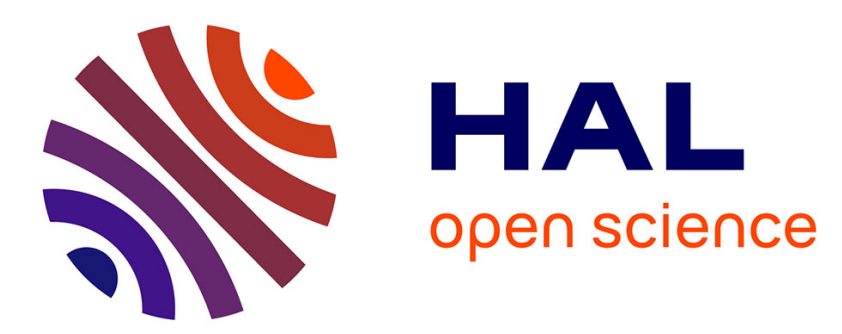

\title{
Decentralized optimization of energy exchanges in an electricity microgrid
}

\author{
Benoît Vinot, Florent Cadoux, Rodolphe Héliot
}

\section{To cite this version:}

Benoît Vinot, Florent Cadoux, Rodolphe Héliot. Decentralized optimization of energy exchanges in an electricity microgrid. 2016 IEEE PES Innovative Smart Grid Technologies Conference Europe (ISGTEurope), Oct 2016, Ljubljana, Slovenia. pp.1-6, 10.1109/ISGTEurope.2016.7856319 . hal-01784148

\author{
HAL Id: hal-01784148 \\ https://hal.inria.fr/hal-01784148
}

Submitted on 3 May 2018

HAL is a multi-disciplinary open access archive for the deposit and dissemination of scientific research documents, whether they are published or not. The documents may come from teaching and research institutions in France or abroad, or from public or private research centers.
L'archive ouverte pluridisciplinaire HAL, est destinée au dépôt et à la diffusion de documents scientifiques de niveau recherche, publiés ou non, émanant des établissements d'enseignement et de recherche français ou étrangers, des laboratoires publics ou privés. 


\section{Decentralized Optimization of Energy Exchanges in an Electricity Microgrid}

\author{
Benoît Vinot \\ Schneider-Electric and G2ELab \\ 37 quai Paul Louis Merlin \\ 38050 Grenoble Cedex 9 \\ France \\ benoit.vinot@schneider-electric.com
}

\author{
Florent Cadoux \\ G2ELab \\ 21 avenue des Martyrs, CS 90624 \\ 38031 Grenoble Cedex 1 \\ France \\ florent.cadoux@g2elab.grenoble-inp.fr
}

\author{
Rodolphe Héliot \\ Schneider-Electric \\ 37 quai Paul Louis Merlin \\ 38050 Grenoble Cedex 9 \\ France \\ rodolphe.heliot@schneider-electric.com
}

\begin{abstract}
We propose to control a local electricity grid, a "microgrid", in a decentralized fashion to reduce energy costs by coordinating the generation and consumption decisions made by the individual Distributed Energy Resources (DERs) composing the system. Our control scheme relies on the so-called Alternative Direction Method of Multipliers or ADMM, the essentials of which we summarize in the first part of the paper. Then we describe our models for loads, batteries, solar panels and wires before investigating different scenarios in which our coordination scheme may be more beneficial than independent decisionmaking. The first scenario demonstrates how our coordinated control strategy may reduce total expenses under variable tariffs of energy. Since this first method does not, however, ensure a gain for each user of the microgrid individually, our second scenario explores a slightly different control strategy designed to ensure an individual benefit to each user, at the cost of decreasing the total benefit.
\end{abstract}

Index Terms-Smart grids, Micro grids, Energy management, Load management, Power Flow.

\section{MATHEMATICAL NOTATIONS}

- For all $(n, m) \in \mathbb{Z} \times \mathbb{N}, \llbracket n, n+m \rrbracket$ denotes the set of integers $\{n, n+1, \ldots, n+m\}$.

- $\mathbb{N}^{*}$ denotes the set of positive integers,

- For all $(n, m) \in\left(\mathbb{N}^{*}\right)^{2}, \mathcal{M}_{n, m}(\mathbb{R})$ denotes the set of real matrices of $n$ rows and $m$ columns.

- For all $n \in \mathbb{N}^{*}, \Phi_{n}$ denotes the lower triangular matrix whose non-zero elements are all ones.

- For all $n \in \mathbb{N}^{*}, \mathbf{0}_{n}$ denotes the zero vector of $\mathbb{R}^{n}$.

- For all $n \in \mathbb{N}^{*}, \mathbf{1}_{n}$ denotes the vector of all ones of $\mathbb{R}^{n}$.

- For all set $\mathcal{E},|\mathcal{E}|$ denotes its cardinal.

\section{INTRODUCTION}

Increasing costs of energy, proliferation of electrical appliances, and climate change are major drivers that are reshaping the power generation, distribution, and usage landscape. Information and Communication Technologies have already proven useful to tackle the challenges ahead, leading to the idea of a Smart Grid. The Smart Grid can bring multiple benefits such as a more efficient transmission of electricity, better resilience, lower power costs for consumers, etc.

It will take time before the electrical grid is fully transformed. During that transition period, pilot projects are needed to demonstrate the effectiveness of upcoming technologies on a local scale before they may be deployed on a larger scale. Microgrids, defined as local, interconnected energy systems, are thus well suited to play this role; typical examples of microgrids include islands, remote communities, large facilities, campuses, municipalities, etc. They incorporate Distributed Energy Resources (DERs), such as power generation, renewables, controllable loads, storage, etc. They can be connected to the main grid, or be operated in islanded mode. As a single entity, a microgrid has its own independent control, which allows testing of advanced control strategies.

It is the role of the Microgrid Energy Management System (MEMS) to balance energy sourcing and consumption while minimizing costs and risks of disturbances such as an outage.

Many ideas were suggested already to solve this issue in a distributed fashion. One of the most widespread is to use the very general framework of multi-agent systems as it has been done at the microgrid level in [1] with a market-based control, or in [2] with an artificial neural network as learning module, or in [3] with a system of auctions. Multi-agents systems were also used at the building level [4], [5]. Although the exact logic of a multi-agent control scheme varies widely from one author to the other, it may be stated that these schemes generally suffer from the lack of mathematical convergence proofs, that translates into lack of guarantees concerning the solution quality as well as the convergence speed.

Among other methods, multi-level optimization is a promising solution and has successfully been used at microgrid level [6] and at building level [7]. Dual decomposition is also an interesting alternative [8], [9]. Those methods are not fully distributed in the sense that they still require a central controller that consolidates the results from, and dispatches commands to, its subsystems.

In this article, we propose to use the "Alternative Direction Method of Multipliers" (ADMM), in order to optimize the energy bill of a microgrid. This method was already used in order to compute optimal schedule in an electrical network [10] or in [11] for electric vehicles charging. Here, we show that this method more generally provides a generic way to coordinate producers and consumers in a microgrid in a decentralized manner. Contrary to most multi-agent schemes, the method relies on solid mathematical foundations; and as 
opposed to multi-level optimization and dual decomposition, it is fully distributed and does not imply any centralized controller anywhere in the system.

The paper is organized as follow: section II provides an overview of the optimization method (ADMM), section III describes the devices that will compose our microgrids and their mathematical model, and in section IV we finally apply our method to several illustrative scenarios to demonstrate its key features and capabilities.

\section{FRAMEWORK}

This section provides the mathematical background that underlies the method we use in the sequel to minimize the total cost of energy in a microgrid over a given horizon of time. We use the ADMM which has recently been used for this kind of purpose by Kraning et al. in [10]. Whenever the necessary mathematical assumptions are met [12], this method may be used to perform all computations in a distributed fashion.

\section{A. Modelling a District as a Network}

Here, for the sake of clarity, we use the same notations as in the pioneering work of Kraning [10]. We define a network as a finite set of terminals, $\mathcal{T}$, a finite set of devices, $\mathcal{D}$, and a finite set of nets, $\mathcal{N}$. Devices are elements of the modelled microgrid such as batteries, buildings, wires, etc. Nets must be considered as exchange zones of electrical power between devices. And lastly, terminals are crossing points which allow power to move from a device to a net or vice-versa.

Formally, devices are connected to nets by terminals so that $\mathcal{D}$ and $\mathcal{N}$ are both partitions of $\mathcal{T}$. For all devices $d \in \mathcal{D}$ and for all terminals $t \in \mathcal{T}$ connected to $d$, we note $t \in d$ (such that the letter " $d$ " denotes both the device itself and its set of terminals). In the same way, for all nets $n \in \mathcal{N}$ and for all $t \in \mathcal{T}$ connected to $n$, we note $t \in n$.

For all terminals $t \in \mathcal{T}$, the goal of the method is to compute the power schedule $p_{t}=\left(p_{t}(1), p_{t}(2), \ldots, p_{t}(T)\right)$ over a given horizon of $T \in \mathbb{N}^{*}$ time-steps. This schedule results from an optimization process taking into account:

- the objective and internal constraints of each individual device, and

- coupling constraints stating that power exchanged between devices is conserved.

To shorten notations, for all $d \in \mathcal{D}$, the vector $p_{d} \in \mathbb{R}^{T \times|d|}$ denotes $\left(p_{t}\right)_{t \in d}$ and for all $n \in \mathcal{N}$, the vector $p_{n} \in \mathbb{R}^{T \times|n|}$ denotes $\left(p_{t}\right)_{t \in n}$.

\section{B. ADMM Principle}

At each iteration $k \in \mathbb{N}^{*}$ of ADMM, each device first solves an optimization problem including its own objective and constraints, and an additional term that relates to neighbouring devices. We refer the reader to [10], [12] for details. The problem formulation can be summarized by the following equation for all $d \in \mathcal{D}$ :

$$
p_{d}^{k}=\underset{p_{d} \in \mathcal{C}_{d}}{\operatorname{argmin}}\left(f_{d}\left(p_{d}\right)+\frac{\rho}{2}\left\|p_{d}^{k-1}-{\overline{p_{d}}}^{k-1}-u_{d}^{k-1}-p_{d}\right\|_{2}^{2}\right)
$$

where $f_{d}$ is the local objective function of the device, $\mathcal{C}_{d}$ is its set of constraints and $p_{d}$ is its power schedule. We also define $\overline{p_{d}}$ and $u_{d}$ as the average power imbalance and the incentive received by the various nets a particular device is connected to.

From this solution, we extract the schedule of power consumed or produced by terminals of each device for the next horizon of $T$ time-steps $i e$. for all $d \in \mathcal{D}$, we have $\left(p_{t}^{k}\right)_{t \in d}$. Then, devices communicate their power schedule to the nets they are connected to, so that all nets $n \in \mathcal{N}$ can compute their average power imbalance :

$$
{\overline{p_{n}}}^{k}=\frac{1}{|n|} \sum_{t \in n} p_{t}^{k} \in \mathbb{R}^{T}
$$

and their local incentive :

$$
u_{n}^{k}=u_{n}^{k-1}+{\overline{p_{n}}}^{k} .
$$

Second, nets communicate their average power imbalance $\overline{p_{n}}$ and their local incentive $u_{n}$ to devices they are connected to. Intuitively, the role of these two terms is to orientate the local optimization which will be performed by devices at the next ADMM step, in order to force nets be conservative power exchangers: for all $n \in \mathcal{N}, \overline{p_{n}}=\mathbf{0}_{T}$ is indeed required at the end of the optimization process. This ensures the physical feasibility of the power consumption/production schedule on the time horizon.

One step of these exchanges is detailed in Figure 1 for a single net.

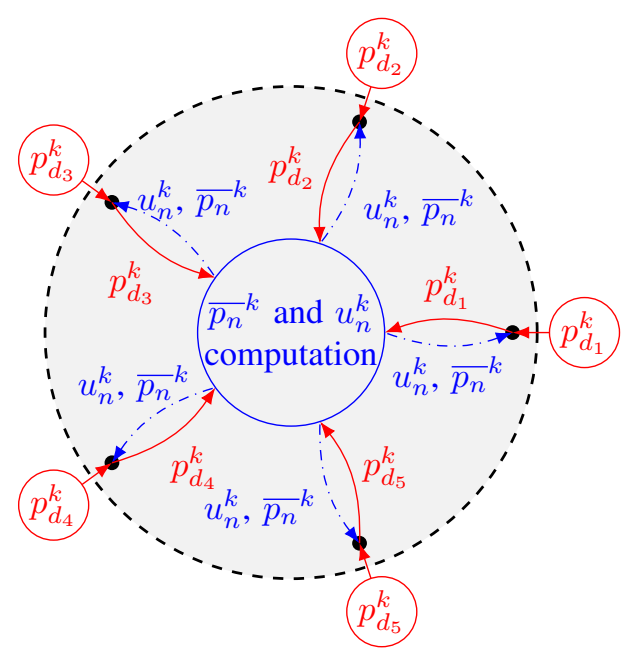

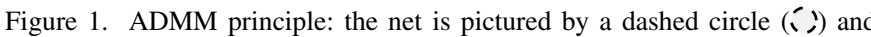
terminals are pictured by black dots $(\bullet)$. Red solid arrows correspond to the first sub-step ( $p_{d}^{k}$ computation and transmission) and blue dashed/dotted arrows correspond to the second sub-step (average power imbalance and local incentive computation and transmission) of an ADMM step.

\section{MODELS}

Let us now introduce models of devices which will be later used. Each of them is modelled as an optimization problem with its own objective and constraints. The battery and the building models are fully described. For other models, the main cost $f_{d}$ and the constraints are given. 
Table I

MODELS' PARAMETERS

\begin{tabular}{|c|c|c|c|}
\hline Name & Description & Unit & Domain \\
\hline \multicolumn{4}{|c|}{ Battery } \\
\hline$P^{+}$ & Maximum power input & $\mathrm{kW}$ & $\mathbb{R}^{+*}$ \\
\hline$P^{-}$ & Maximum power output & $\mathrm{kW}$ & $\mathbb{R}^{+*}$ \\
\hline$\underline{b}$ & Minimal state of charge & $\mathrm{kWh}$ & $\mathbb{R}^{+*}$ \\
\hline$\overline{\bar{b}}$ & Maximal state of charge & $\mathrm{kWh}$ & $(\underline{b},+\infty)$ \\
\hline$b_{0}$ & Initial state of charge & $\mathrm{kWh}$ & {$[\underline{b}, \bar{b}]$} \\
\hline$\eta^{+}$ & Charge efficiency & - & $(0,1]$ \\
\hline$\eta^{-}$ & Discharge efficiency & 一 & $(0,1]$ \\
\hline \multicolumn{4}{|c|}{ Grid Connection } \\
\hline$P_{\mathrm{r}, \max }$ & Maximum output power & $\mathrm{kW}$ & $\mathbb{R}^{+}$ \\
\hline$P_{\mathrm{p}, \max }$ & Maximum input power & $\mathrm{kW}$ & $\mathbb{R}^{+}$ \\
\hline$\Gamma_{\mathrm{r}}$ & Electricity resale price & $€ \cdot \mathrm{kWh}^{-1}$ & $\mathbb{R}^{T}$ \\
\hline$\Gamma_{\mathrm{p}}$ & Electricity purchase price & $€ \cdot \mathrm{kWh}^{-1}$ & $\mathbb{R}^{T}$ \\
\hline$E_{\max }$ & Maximum allowed expenses & $€$ & $\mathbb{R}$ \\
\hline \multicolumn{4}{|c|}{ Solar Panel } \\
\hline$P_{\max }$ & Maximum producible power & $\mathrm{kW}$ & $\left(\mathbb{R}^{+}\right)^{T}$ \\
\hline$\Gamma$ & $\begin{array}{c}\text { Penalty for non produced } \\
\text { energy }\end{array}$ & $€ \cdot \mathrm{kWh}^{-1}$ & $\mathbb{R}^{T}$ \\
\hline
\end{tabular}

\section{A. Battery}

A battery is a single terminal device whose parameters are gathered in Table I. Its power schedule $p \in \mathbb{R}^{T}$ is split into its positive and negative components $p^{+}=\max (0, p) \in\left(\mathbb{R}^{+}\right)^{T}$ and $p^{-}=\min (0, p) \in\left(\mathbb{R}^{-}\right)^{T}$ such that $p=p^{+}+p^{-}$.

At iteration $k \in \mathbb{N}^{*}$ of ADMM, the optimization problem of a battery is:

$$
\left\{\begin{array}{c}
\min _{p^{+}, p^{-}} \frac{\rho}{2}\left\|p^{k-1}-{\overline{p_{n}}}^{k-1}-u_{n}^{k-1}-\left(p^{+}+p^{-}\right)\right\|_{2}^{2} \\
\left(\begin{array}{c}
\mathbf{0}_{T} \\
-P^{-} \mathbf{1}_{T}
\end{array}\right) \leqslant\left(\begin{array}{c}
p^{+} \\
p^{-}
\end{array}\right) \leqslant\left(\begin{array}{c}
P^{+} \mathbf{1}_{T} \\
\mathbf{0}_{T}
\end{array}\right) \\
\left(\underline{b}-b_{0}\right) \mathbf{1}_{T} \leqslant \tau\left(\eta^{+} \Phi_{T} \mid \frac{1}{\eta^{-}} \Phi_{T}\right) \\
\left.\quad \begin{array}{l}
p^{+} \\
p^{-}
\end{array}\right) \\
\forall t \in \llbracket\left(\bar{b}-b_{0}\right) \mathbf{1}_{T} \\
\forall 1, T \rrbracket, p^{+}(t) p^{-}(t)=0 .
\end{array}\right.
$$

From this point, $\rho \in \mathbb{R}^{+}$is a mathematical parameter in $€ \cdot \mathrm{kWh}^{-2}, \tau \in \mathbb{R}^{+*}$ is the time-step of our models expressed in hours and for single terminal devices, $n \in \mathcal{N}$ is the net to which the device is connected through its single terminal. The value of $\rho$ only influences the convergence speed of the algorithm and will not be considered further in this paper, both due to space limitation and because our focus here is on the application of the ADMM to power grid problems and not on the internal working of the ADMM itself.

The objective function (1) of the battery reduces to (4a), which reflects the fact that this device essentially does not imply any operational cost. The battery has access to $p^{k-1}$ which is its power schedule of the previous ADMM iteration. ${\overline{p_{n}}}^{k-1}$ and $u_{n}^{k-1}$ are computed by the net the battery is connected to through its single terminal. Constraint (4b) ensures that the power input and output $\left(p^{+}, p^{-}\right)$are restricted to the range $\left[0, P^{+}\right]$and $\left[-P^{-}, 0\right]$ respectively. Constraint $(4 \mathrm{c})$ limits the state of charge between the minimum and the maximum allowed states of charge. Finally, constraint (4d) prevents battery from charging and discharging at the same time (and, mathematically speaking, is optional).

\section{B. Grid Connection}

A grid connection is a single terminal device which is able to sell and to purchase electricity. Similarly to what was done for the battery, we introduce $p^{+}=\max (0, p) \in\left(\mathbb{R}^{+}\right)^{T}$ and $p^{-}=\min (0, p) \in\left(\mathbb{R}^{-}\right)^{T}$ the purchased power and the opposite of the produced power.

Its cost function is $f_{d}\left(p^{+}, p^{-}\right)=\tau\left[\Gamma_{\mathrm{r}} p^{-}+\Gamma_{\mathrm{p}} p^{+}\right]$and the constraints are the following: the total expenses are limited by $E_{\max }$ and the purchase and resale powers are respectively limited by $P_{\mathrm{p}, \max }$ and $P_{\mathrm{r}, \max }$.

\section{Wire}

A wire is a two-terminal device; their respective power schedules are denoted by $p_{1} \in \mathbb{R}^{T}$ and $p_{2} \in \mathbb{R}^{T}$. We also use the same subscripts to denote all quantities which come from the first or the second terminal respectively eg. $n_{1}$ for the net to which the first terminal belongs.

The objective function of this device is zero and its constraints impose that electricity is carried from a terminal to the other in a conservative way so $p_{1}+p_{2}=\mathbf{0}_{T}$.

From this point, there are two ways to apprehend a "wire":

- it can be viewed as a real wire in an electrical network. In this case, we can limit its capacity to a maximum $C$ expressed in $\mathrm{kW}$;

- or it can be used as a purely financial exchange in a cooperative network of electricity consumers, assuming that the underlying physical grid has sufficient capacity to accommodate all flows that make financial sense. In this case, $C$ is simply ignored.

\section{Solar Panel}

A solar panel is a single terminal device whose available power is defined by the solar irradiance. However, we let it the possibility to curtail this maximum theoretical production if necessary. Its objective function is $f_{d}(p)=\tau \Gamma\left(p+P_{\max }\right)$ and the constraints limit the maximum production and curtailment.

The parameter $\Gamma$ can be viewed as a shortfall (loss of revenue) for non-produced energy: that is why it is expressed as a price.

\section{E. Building}

A building is a single terminal device whose objective is to minimize its operational cost under some comfort constraints such as temperature, $\mathrm{CO}_{2}$ concentration, etc. We use the building model described in [7]. We present here the main points of the optimization process: variables of the optimization problem are $z$, which gathers every decision variables of the building including the power consumption schedule, and $y$, which gathers the outputs (temperature, $\mathrm{CO}_{2}$ concentration and brightness) of the building's simulation. So there exists a matrix $E$ such that $E z=p \in \mathbb{R}^{T}$ where $p$ is the power consumption schedule. 
In order to solve the problem described in [7], a fixed-point algorithm is used. Let $k \in \mathbb{N}^{*}$ be the iteration number of the ADMM process. For each ADMM step, we note $l \in \mathbb{N}^{*}$ the number of the current fixed-point algorithm iteration. We begin to solve:

$$
\left\{\begin{array}{c}
\min _{z^{k, l}} L(\tau, \Gamma) z^{k, l} \\
\quad+\frac{\rho}{2}\left\|p^{k-1}-{\overline{p_{n}}}^{k-1}-u_{n}^{k-1}-E z^{k, l}\right\|_{2}^{2} \\
\underline{z} \leqslant z^{k, l} \leqslant \bar{z} \\
A\left(y^{k, l}\right) z^{k, l} \leqslant b
\end{array}\right.
$$

where:

- $\Gamma$ is the electricity price expressed in $€ \cdot \mathrm{kWh}^{-1}$,

- $L(\tau, \Gamma)$ is a vector which depends on $\tau$ and $\Gamma$,

- $A(y)$ is a matrix which depends on $y$,

- $b, \underline{z}, \bar{z}$ are vectors,

- $\mathcal{Z}$ is a non-linear operator which simulates zones in the building. Its inputs are $z$ which is the vector of decision variables, $w$ which is a given vector of perturbations and $i$, which is the initial state of zones. This operator also includes specific properties of the building so it differs from one building to another.

and after, we inject the result $z^{k, l}$ :

$$
y^{k, l+1}=\mathcal{Z}\left(z^{k, l}, w, i\right) .
$$

We can then solve (5) with $y^{k, l+1}$ and so on until convergence of the fixed-point algorithm.

\section{Simulation Scenarios and Results}

\section{A. District and customers}

First, let us define a client in our microgrid. A client is modelled by a net which is connected to all its "equipments": eg. in Figure 2, the "orange disc" 5 depicts a client who owns and manages a building, solar panels and a grid connection. Moreover, this client is connected to two other clients $(5,5)$ with wires.

\section{B. Scenario 1: Identifying Network Potentialities}

Our first purpose is to demonstrate how clients, as a whole, may save money by coordinating their actions. To do so, let us consider the network in Figure 2 and its parameters in Table II. Here, wires are only considered as financial link between clients as it has been discussed in subsection III-C, thus $C=\infty$ (no physical limit is introduced within the microgrid). Furthermore, the electricity price for internal exchanges (meaning, exchanges between two buildings of the same microgrid through "wires") is set to $\mathbf{0}_{T}$; electricity is only billed when it enters the microgrid from the outside world through external grid connections (terminals $\bigcirc$ in Figure 2).

The ADMM parameter $\rho$ is set at 1 , the time-step at $20 \mathrm{~min}$ and the horizon schedule at $48 \mathrm{~h}$ (so $T=144$ ). The simulation protocol is the following.

- First, clients are considered as independent; a simulation is performed as if wires were not present, meaning there is no cooperation between clients. The final bill paid to

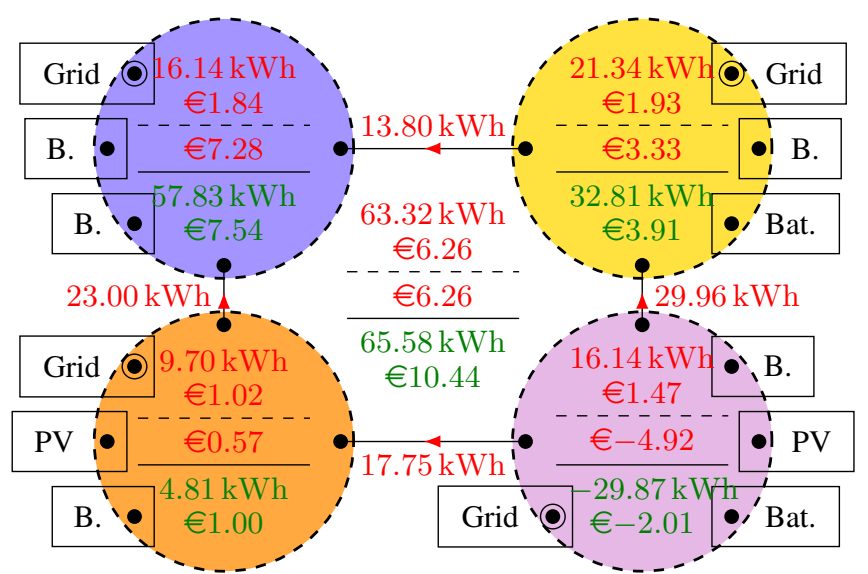

Figure 2. Network 1. Consumptions/expenses balances of clients alone are written in green below solid lines. Their consumption/expenses when they are connected are written in red without compensations for exchanged energy above dashed lines and with compensations at grid resale price below dashed lines. "B." stands for "Building" and "Bat." for "Battery".

the grid ie. the cost of purchased electricity minus the revenue generated by electricity sales, is displayed in green in Figure 2.

- Then, we add wires and run the simulation again; results are displayed in red above solid lines.

Table II

\begin{tabular}{|c|c|c|}
\hline \multicolumn{3}{|c|}{ Identical devices for all nets } \\
\hline \multirow{4}{*}{ Grid Connections } & $P_{\mathrm{p}, \max }=P_{\mathrm{r}, \max }$ & $5 \mathrm{~kW}$ \\
\hline & 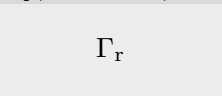 & $\begin{array}{c}0.14 € \cdot \mathrm{kWh}^{-1} \text { from } \\
\text { 6:00am to } 10: 00 \mathrm{pm}, \\
0.09 € \cdot \mathrm{kWh}^{-1} \text { otherwise }\end{array}$ \\
\hline & $\Gamma_{\mathrm{p}}$ & $\frac{1}{2} \Gamma_{\mathrm{r}}$ \\
\hline & $E_{\max }$ & $\infty$ \\
\hline Buildings & $\Gamma$ & $\mathbf{0}_{T} € \cdot \mathrm{kWh}^{-1}$ \\
\hline Wires & $C$ & $\infty$ \\
\hline \multicolumn{3}{|c|}{ Net 2} \\
\hline \multirow{4}{*}{ Battery } & $P^{+}=P^{-}$ & $5 \mathrm{~kW}$ \\
\hline & $b_{0}=\underline{b}$ & $0 \mathrm{kWh}$ \\
\hline & $\bar{b}$ & $15 \mathrm{kWh}$ \\
\hline & $\eta^{+}=\eta^{-}$ & 0.95 \\
\hline \multicolumn{3}{|c|}{ Net 3} \\
\hline \multirow[t]{2}{*}{ Solar Panels } & $P_{\max }$ & $\begin{array}{l}\text { Gaussian curve with a pro- } \\
\text { duction peak of } 2 \mathrm{~kW} \text { at } \\
\text { 12:00 Noon and a standard } \\
\text { deviation of } 3 \text { hours. }\end{array}$ \\
\hline & $\Gamma$ & $\begin{array}{l}\text { Twice the resale price of a } \\
\text { grid connection. }\end{array}$ \\
\hline \multicolumn{3}{|c|}{ Net 4} \\
\hline \multirow{4}{*}{ Battery } & $P^{+}=P^{-}$ & $5 \mathrm{~kW}$ \\
\hline & $b_{0}=\underline{b}$ & $0 \mathrm{kWh}$ \\
\hline & $\bar{b}$ & $10 \mathrm{kWh}$ \\
\hline & $\eta^{+}=\eta^{-}$ & 0.95 \\
\hline \multirow[t]{2}{*}{ Solar Panels } & $P_{\max }$ & $\begin{array}{l}\text { Gaussian curve with a pro- } \\
\text { duction peak of } 4 \mathrm{~kW} \text { at } \\
2: 00 \mathrm{pm} \text { and a standard de- } \\
\text { viation of } 3 \text { hours. }\end{array}$ \\
\hline & $\Gamma$ & $\begin{array}{l}\text { Twice the resale price of a } \\
\text { grid connection. }\end{array}$ \\
\hline
\end{tabular}

PARAMETERS FOR SimUlations OF NETWORK \#1 
One can remark that total expenses at the microgrid level decrease, while the same level of comfort has been guaranteed to buildings. Solar panels produce the maximum they can in both simulations because a sharp penalty is applied, so the first reason of this improvement is due to batteries: alone, clients with batteries do not use them at their maximum potential because they have no interest to store electricity which they do not use within a day. Briefly, they store the energy they need for an entire day during off-peak hours and they give back this energy during on-peak hours. But, when clients work together, batteries store energy for all clients and are then used at their maximum potential.

The second reason of this improvement is due to the use of solar energy: when clients are alone, and when solar panels produce energy they can not consume, they sell it to the grid at low cost (see Table II, parameter $\Gamma_{\mathrm{p}}$ ). When they are connected, this energy can be used by other clients, avoiding them the need to purchase from the grid at a higher price than the power resale price.

Let us now consider individual gains. The first cost (in red above dashed lines in Figure 2) is the direct result of the optimization process. Here, we notice that the most flexible clients (that is to say, those with solar panels and/or batteries) $(5,5)$ are not those who earn the most, on the contrary! The reason is that services they provide (energy storage and/or solar energy provider) are simply not paid ie. they supply energy freely to other, less flexible members of the microgrid.

In order to take into account these services, a postoptimization compensation of the energy exchanged between clients through wires may be performed. For instance, compensating internal exchanges at price $\Gamma_{r}$ (ie. the same price as for reselling energy to the external grid) yields the second cost (in red below dashed lines in Figure 2). As a result, clients without flexibility now make no or very little gain (5) and the benefits is essentially transferred to more flexible players. This phenomenon is shown in Figure 3.

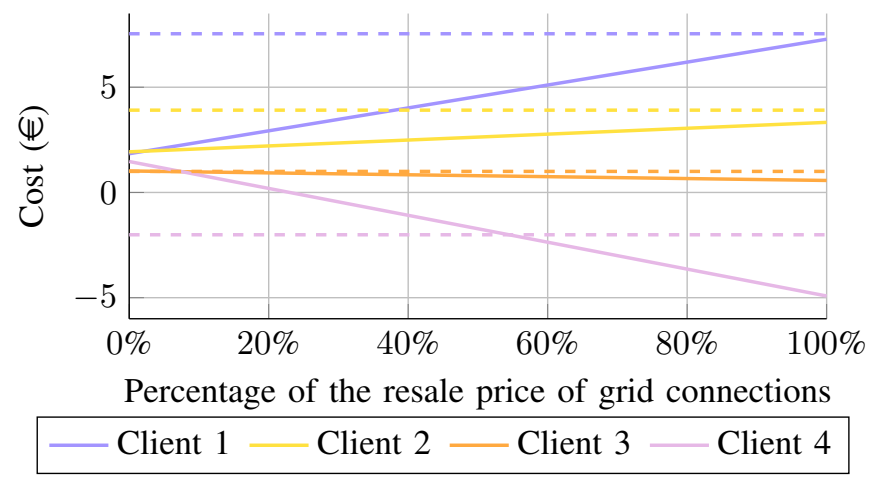

Figure 3. Clients' expenses according to the valuation of exchanges. Dashed lines are the results when clients act alone.

For instance, client $4(1)$, who is the most flexible loses money when it is connected to the network if there are no compensations and client $1(5)$, who is the least flexible, earns a lot. On the opposite, if compensations are done at grid resale price, client 4 earns a lot and client 1 does not earn money any more.

This example illustrates how make-whole payments may be used to distribute common benefits, resulting from coordination, to individual players in such a way that non-negative individual gains are obtained for all, and flexible clients are rewarded for their contribution. In this example, this was achieved by using — quite arbitrarily — the external grid resell price as an internal price. The upside of this method is that it simply redistributes the total gain in a fairer way, without reducing this total. One of its drawbacks is that it requires potentially complex ex-post financial settlements involving all players, somehow losing the benefit of using a fully decentralized control method. We now consider a more systematic, and distributed way, of reaching the same result.

\section{Scenario 2: Ensuring Gain}

In order to ensure a gain for everybody, a technical solution can be found: introducing constraints. Here are the main steps of the method:

- As it has been done in scenario 1 , each client is first optimized independently with the ADMM by "deleting" wires. These results are written in green in Figure 4.

- Next, the entire microgrid (with wires) is optimized with the ADMM and we use the parameter $E_{\max }$ of grid connections in order to restrict clients' expenses to expenses they reach alone. So, the grid connection of the first client (1) can not spend more than $€ 7.54$, the one of the second (1) can not spend more than $€ 3.91$, etc. Results of this optimization are written in blue above dashed lines in Figure 4. Results with post-optimization compensations for the energy exchanged through wires at grid connections resale price are written in blue below dashed lines.

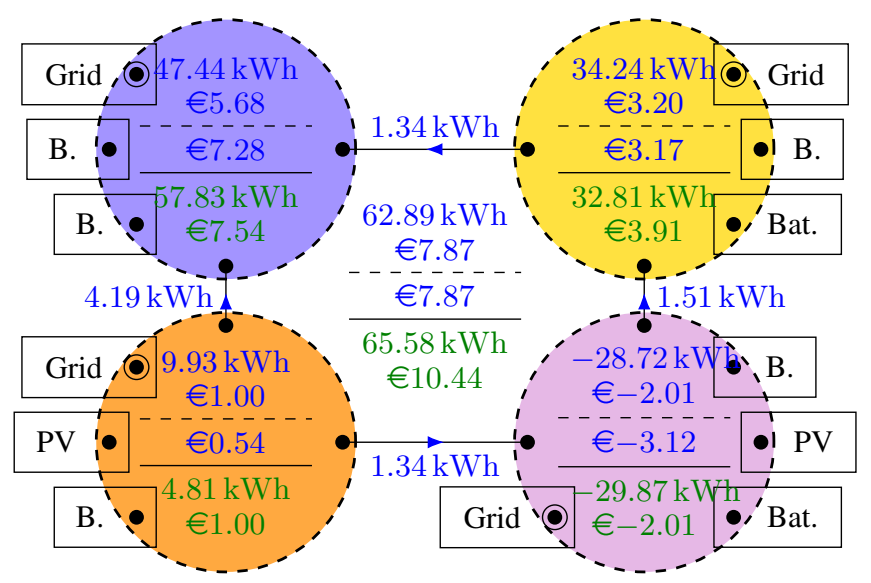

Figure 4. Network 1 bis: here expenses of grid connections have been limited with their parameter $E_{\max }$. Results in green below solid lines are for clients alone. In blue, above dashed lines, results without postoptimization compensations for exchanged energy and below dashed lines with compensations at grid resale price. "B." stands for for "Building" and "Bat." for "Battery". 
We can first check that financial constraints have been respected: each client does not spend more than if it were alone. Second, we notice that even if financial constraints have been added, the problem still has a solution because the "freefor-all" solution, which has been found at the first step of the process, exists and does not violate the new constraints. In fact, the solution found with the connected optimization, as before, can not be globally worse.

But, even if a solution exists, it is sub-optimal compared with the one found without financial restrictions: $€ 7.87$ against $€ 6.26$ previously reached. This is the cost to ensure gain for every client with this technical solution: adding constraints that enforce a certain "fairness" unfortunately degrades the global optimum.

\section{CONCLUSION}

The above simulations were performed to illustrate the flexibility of the ADMM and its ability to perform:

- fully decentralized control of an electricity microgrid,

- on a wide variety of test cases,

- using a method with solid mathematical foundations

The first feature, full decentralization, is seen as beneficial since decentralized approaches scale up well, and are more resilient than centralized methods based on a single controller.

The second feature, genericity, is important because largescale deployment of smart grid technologies calls for generic control schemes that may be applied to a wide range of situations without local fine-tuning. We showed how a generic optimization method without any preset specific decision rules, applied to a wide range of situations, and may uncover gain opportunities as varied as:

- using a player's batteries for the benefit of other members of the microgrid;

- reselling power internally rather than to the external grid to benefit from a price gap between buy- and resell-prices;

- deferring load in time to better match times of high solar generation;

- coordinating local loads to overcome a common capacity limit on a wire (or, similarly, a limited contracted power).

The third feature, being based on solid mathematical theory, paves the way for fully automated control methods that will provide guaranteed functionality and, again, have the potential to scale up without failures.

The key assumption that allows such a dynamic management of microgrids is the availability of flexible devices such as batteries and thermal storages (here built-in inside the models used to manage entire buildings). When such devices are available, optimization methods make it possible to coordinate all members of a microgrid so as to generate global financial gains; and this paper also discussed how these global gains may translate into guaranteed benefits for individual players, at the cost of degrading the global optimum.

This work may be extended in several ways. First, better methods to ensure individual gains may be suggested, especially if such methods lead to a lower degradation of social gains or provide provably sound economic incentives for investment in flexible devices such as batteries, solar panels, electric vehicles with deferrable charging, etc. Second, only the point of view of the end-user was taken into account in this work; it is thus important to study how other players, such as suppliers and Distribution System Operators (DSOs), would fit into our framework, and adapt to the new behaviour of their loads when these loads start exchanging power in a bilateral way. Finally, and assuming a much better modelling of the underlying physics of power systems, this work could be extended to the management of low-level physical constraints on the electricity grid such as voltage and current constraints. These improvements are left as matter for future research.

\section{REFERENCES}

[1] K. Kok, G. Venekamp, and P. Macdougall, "Market-based control in decentralized electrical power systems", in First international workshop on agent technologies for energy systems, ATES2010, toronto, 2010.

[2] L. Gomes, P. Faria, H. Morais, Z. Vale, and C. Ramos, "Distributed, agent-based intelligent system for demand response program simulation in smart grids", IEEE intelligent systems, vol. 29, no. 1, pp. 56-65, Jan. 2014, ISSN: 1541-1672.

[3] N. Capodieci, G. Cabri, G. Pagani, and M. Aiello, "An agentbased application to enable deregulated energy markets", in Computer software and applications conference (COMPSAC), 2012 IEEE 36th annual, Jul. 2012, pp. 638-647.

[4] S. Abras, "Système domotique multi-agents pour la gestion de l'énergie dans l'habitat", PhD thesis, Grenoble, INPG, Jan. 1, 2009.

[5] S. Smitha, J. Savier, and F. Chacko, "Intelligent control system for efficient energy management in commercial buildings", in 2013 annual international conference on emerging research areas and 2013 international conference on microelectronics, communications and renewable energy (AICERA/ICMiCR), Jun. 2013, pp. 1-6.

[6] D. L. Ha, F. de Lamotte, and Q.-H. Huynh, "Real-time dynamic multilevel optimization for demand-side load management", in 2007 IEEE international conference on industrial engineering and engineering management, Dec. 2007, pp. 945-949.

[7] M. Y. Lamoudi, "Commande prédictive distribuée pour la gestion de l'énergie dans le bâtiment", $\mathrm{PhD}$ thesis, Université de Grenoble, Nov. 29, 2012.

[8] B. Biegel, J. Stoustrup, and P. Andersen, "Distributed model predictive control via dual decomposition", Intelligent systems, control and automation: Science and engineering, vol. 69, 2013.

[9] P. Pflaum, M. Alamir, and M. Lamoudi, "Comparison of a primal and a dual decomposition for distributed MPC in smart districts", in 2014 IEEE international conference on smart grid communications (SmartGridComm), Nov. 2014, pp. 55-60.

[10] M. Kraning, E. Chu, J. Lavaei, and S. Boyd, "Dynamic network energy management via proximal message passing", Foundations and trends in optimization, vol. 1, no. 2, pp. 1-54, 2013.

[11] W.-J. Ma, V. Gupta, and U. Topcu, "On distributed charging control of electric vehicles with power network capacity constraints", American control conference, 2014.

[12] S. Boyd, N. Parikh, E. Chu, B. Peleato, and J. Eckstein, "Distributed optimization and statistical learning via the alternating direction method of multipliers", Foundations and trendsó in machine learning, vol. 3, no. 1, pp. 1-122, 2011. 